\title{
Inflammatory bowel disease: Why do I have these symptoms?
}

\author{
S LEMIRE, MD, FACG
}

W HETHER A PATIENT HAS SYMPtoms of inflammatory bowel disease (IBD) is dependent on the activity and the extent of involvement. Patients in quiescent states may have no symptoms at all, while, during exacerbations, they may be severely ill.

Most people with IBD, irrespective whether they suffer from Crohn's disease or ulcerative colitis, experience three major symptoms: diarrhea, abdominal pain and fever. These are usually associated with weight loss and anemia. The nature of the diarrhea often helps the physician to localize the area of the bowel involved by the inflammatory process.

The type of abdominal pain may also vary depending on the severity and the location of bowel inflammation. Sometimes pain is aggravated by eating. This may lead to decrease intake of food and weight loss. In children, growth may be delayed or retarded. Growth spurts may be stimulated by increasing the daily calorie intake.

Fever is always an important sign of active disease. It may be intermittent or persistent, ranging from $38.5^{\circ}$ to $40^{\circ} \mathrm{C}$, and may be accompanied by shaking chills. Night sweats strongly suggest the presence of an abscess or are a significant clue to the seriousness of the illness.

The causes of anemia in IBD vary from iron deficiency to chronic blood loss, to anemia of chronic disease. Generally speaking, chronic disease depresses the bone marrow and interferes with red blood cell production. Vitamin $B_{12}$ and folic acid deficiency result either from inadequate intake or malabsorption and will lead to megaloblastic anemia.

\section{CROHN'S DISEASE}

Crohn's disease is characterized by ulcers that can involve the whole thickness of the bowel and, on healing, this may result in fibrosis. The disease can affect any portion of the digestive tract from the mouth to the anus. Hence, symptoms will depend on the location of the inflammatory process.

Ulcerations of the mouth can occur and may be painless. Size of these ulcers vary. If there is inflammation in the esophagus, there may be pain with swallowing or heartburn. The stomach may also be affected; the principal symptoms
Laval University, Division of Gastroenterology, Hôpital de l'Enfant-Jésus, Québec, Québec

Correspondence and reprints: Dr Suzanne Lemire, Clinical Professor of Medicine, Laval University, Chief, Division of Gastroenterology, Hôpital de l'Enfant-Jésus, Québec, Québec are nausea, vomiting and upper ahdominal pain. In the duodenum, the symptoms may be similar to those of peptic ulcer: burning or gnawing abdominal pain, nausea and vomiting, The latter two symptoms are due to the impaired passage of food, secondary to inflammation that causes narrowing of the gut adjacent to the stomach.

More typically, Crohn's disease affects segments of the small or the large bowel. If parts of the small bowel are affected, lower abdominal pain may be associated with cramps and bloating after eating, since food stimulates bowel activity.

Inflammation, as stated before, may result in narrowing of the bowel and this may cause partial obstruction. The pain is mostly due to strong contractions and severe spasms of the intestine to overcome the blockage.

When large areas of the small intestine are involved, diarrhea is almost always present. Digestion and absorption of food may be abnormal since the process takes place mostly in the small bowel. Lactose intolerance occurs more frequently in patients with Crohn's disease than in the normal population. Lactase is an enzyme of the small intes. tine that digests lactose in milk and milk products. Lactase deficiency causes bloating and diarrhea. Occasionally, when several loops of the 
small bowel are partially blocked due to inflammation, dilated loops, between narrowed segments, may contain large amounts of bacteria. These bacteria are normally found in the gut. This can also occur if there are abnormal communications - fistulae - between two loops of intestine.

When these bacteria are present in excessive amounts, they may cause malabsorption of fats, since they deconjugate bile acids. This will lead to increased diarrhea. They may also compete with the absorption of nutriments, such as sugar, starches and vitamins, in particular vitamin $\mathrm{B} 12$.

The terminal ileum is the most frequently involved part of the intestinal tract, occuring in $50 \%$ of patients. If the disease is located at the terminal ileum, a tender mass in the right lower quadrant of the abdomen may be present. This mass is often due to inflamed bowel loops that become thick and adherent, or to swelling of surrounding tissues such as the mesentery. Terminal ileal disease results in severe diarrhea for two reasons:

- the inflamed ileum is capable of secreting excessive amounts of water and electrolytes

- bile salts found normally in the small intestine are reabsorbed in this area. If the bowel is inflamed, bile salts will not be absorbed and they will reach the colon in large quantities. Bile salts are known to increase the secretion of water and electrolytes by the colon. This will lead to diarrhea. The electrolytes lost are mostly potassium, sodium, chlorides and bicarbonates.

Vitamin $\mathrm{B}_{12}$ is absorbed at the terminal ileum. When this part of the bowel is diseased, $\mathrm{B}_{12}$ is not absorbed normally and its blood level is decreased. This vitamin is essential for the formation of blood cells. A chronic deficiency will produce anemia.

Crohn's disease may involve the colon. This occurs in $20 \%$ of patients. It may affect the entire colon or only several areas of the large bowel, interposed with normal bowel, producing skip areas. If large areas are involved, water, which would normally be ab. sorbed in this section of the gastrointestinal tract, passes through and causes diarrhea.

Approximately $12 \%$ of people with Crohn's disease have bloody diarrhea and it may be difficult to differentiate them from individuals with ulcerative colitis. Bleeding is due to inflammation and ulcers of the colon. Abscesses and fistulae around the anus occur quite frequently in Crohn's disease, and may even occur before bowel symptoms. In these locations, the abscess is very painful. It may be so painful that patients have problems in walking or sitting. These abscesses occur because of penetrating inflammation from the anal canal or the rectum. Fistulae may occur when there is a communication between a loop of diseased bowel and the perianal area. Fistulae may occur in any area involved with Crohn's disease, such as bowel to bowel, bowel to urinary tract and bowel to skin. This may lead to short circuiting of the bowel, with increased diarrhea, urinary infection and discharging skin lesions.

\section{ULCERATIVE COLITIS}

In ulcerative colitis, inflammation occurs only in the colon and may be limited to the distal part of the colon, the rectum. The latter is called ulcerative proctitis. Since inflammation is on the surface lining the bowel, the first symptoms are bleeding and diarrhea. Severity of symptoms relates to the extent of bowel involvement by inflammation.

When only the rectum is involved by the inflammatory process, which occurs in 25 to $30 \%$ of the ulcerative colitis population, passage of blood or mucus alone may occur several times during the day or night, without any stool. In less severe cases, bowel movements, coated by blood or mucus, are accompanied by formed stools. Mucus is a clear secretion of the digestive tract that may act as a defence mechanism and a lubricant of the intestine. When inflammation occurs, mucus is produced in excessive amount.

When the distal section of the rectum is inflamed, it may lose its capacity to differentiate between liquids and gas. This may lead to incontinence, as patients may pass liquid stools, believing that they are passing flatus.

If a large part of the colon is involved by inflammation, stools become watery and associated with blood and mucus. Patients may pass up to 20 to 30 bowel movements a day.

Diarrhea is often associated with diffuse cramping abdominal pain. The exact cause of pain is uncertain. It can be related to excessive contractions of the bowel musculature, or to the inflammatory process, that irritates pain receptors in the muscles of the bowel wall.

\section{SYMPTOMS OF EXTRAINTESTINAL COMPLICATIONS}

Some individuals with IBD may develop arthritis, which typically occurs in the large joints, usually when the bowel disease is active. Manifestations include joint redness, swelling and fluid accumulation. It is rarely progressive and improves as the bowel disease improves. More unusual forms of arthritis have been associated with IBD, that involve the spine and pelvis. They are called sacroiliitis and ankylosing spondylitis. They occur most frequently in individuals with a particular genetic background and cause low back pain. The evolution does not necessarily correlate with the course of IBD.

Skin problems can occur. Erythema nodosum may develop in 10\% of people. These are tender nodules that appear over the legs and shins. They are reddish-blue, tender, warm and occur more commonly when the disease is active. Pyoderma gangrenosum is a much less common lesion that occurs most frequently on the legs. Eye problems may be associated with IBD. Iritis and uveitis cause pain and redness of the eye. Gallstones, kidney stones and liver abnormalities may develop in some patients. Tests are usually done to disclose these conditions.

Early diagnosis of IBD may be difficult because symptoms can be mild in the first stages. Pain, diarrhea, tenesmus, bloody stools, fatigue and weight loss are often present early in the disease. With any of these symptoms, one must seriously consider the diagnosis of IBD. 
ACKNOWLEDGEMENTS: The author expresses appreciation to Suzanne Bilodeau for assistance in preparing the manuscript.

\section{SUGGESTED READING}

1. Kirsner JB, Shorter RG. Inflammatory Bowel Disease, 3rd edn. Philadelphia: Lea and Febiger, 1988.

2. Both H, Torp-Pedersen K, Kreiner S, Hendriksen C, Binder V. Clinical appearance at diagnosis of ulcerative colitis and Crohn's disease in a regional patient group. Scand J Gastroenterol 1983;18:987-91.

3. Kirsner JB. Local and systemic complications of inflammatory bowel disease. JAMA 1980;242:1177-83.

4. Guido MA, Van Rosendaal GM. Inflammatory bowel disease. Can Med Assoc J 1989;141:113-23.
5. Bagby RJ, Roger JV Jr, Hobbs C. Crohn's disease of the esophagus, stomach and duodenum. South Med J 1972;65:515-23.

6. Ward C, Dunphy EP, Jagoe WS, Sheahan MB. Crohn's disease limited to the mouth and anus. J Clin Gastroenterol 1985;7:516-21.

7. Marshak RH, Maklansky D, Kurzban JD, Lindner AE. Crohn's disease of the stomach and duodenum. Am J Gastroenterol 1982;77:340-3.

8. Nuggent FW, Roy MA. Duodenal Crohn's disease, an analysis of 89 cases. Am J Gastroenterol 1989;84:249-54.

9. Belli DC, Seidman E. Chronic intermittent elemental diet improves growth failure in children with Crohn's disease. Gastroenterology 1988;94:603-10.

10. Schorr-Lesnick B, Brandt LJ. Selected rheumatologic and dermatologic manifestations of inflammatory bowel disease. Am J Gastroenterol 1988;83:216-23.

11. Drossman EA. Inflammatory bowel disease. Dig Dis Sci 1989;34:1379-86.

12. Bockus HL. Gastroenterology, 4 th edn. Philadelphia: WB Saunders, 1985.

13. Kanof ME, Lake AM, Bayless TM. Decreased height velocity in children and adolescents before the diagnosis of Crohn's disease. Gastroenterology 1988;95:1523-7.

14. Michener WM, Greenstreet RL, Farmer RG. Comparison of the clinical features of Crohn's disease and ulcerative colitis with onset in childhood and adolescence. Cleve Clin Q 1983;49:13.

15. Kirsner JB. Inflammatory bowel disease. Its present and its future. Am J Gastroenterol 1989;84:1358-61. 


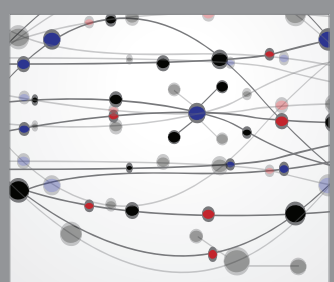

The Scientific World Journal
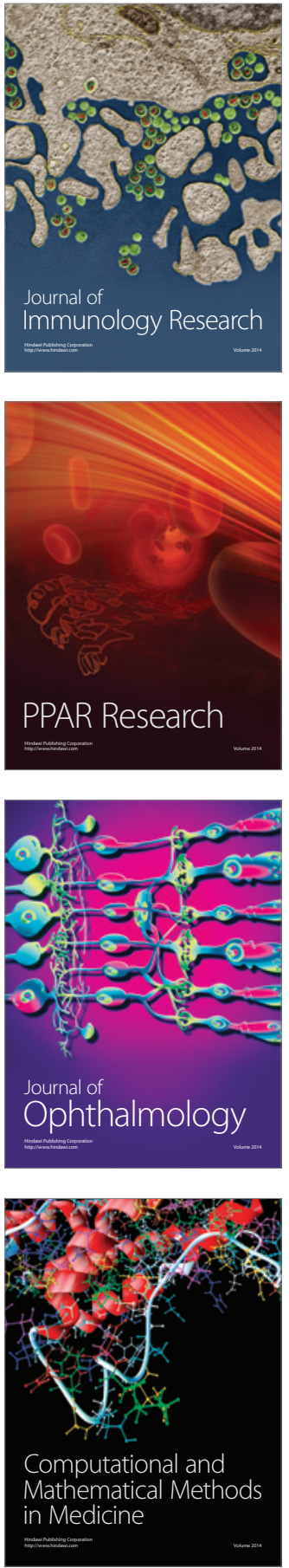

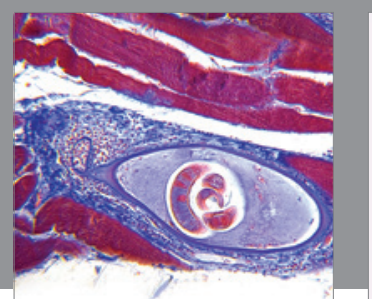

Gastroenterology Research and Practice

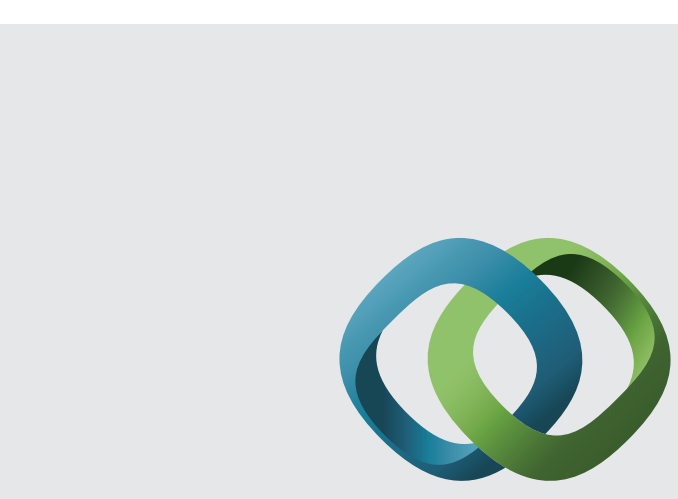

\section{Hindawi}

Submit your manuscripts at

http://www.hindawi.com
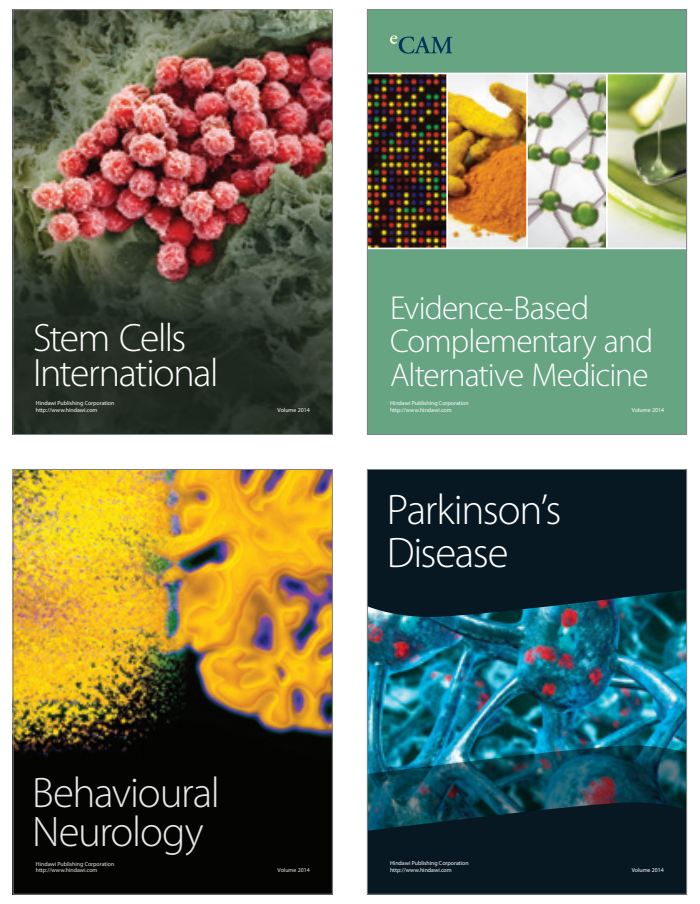
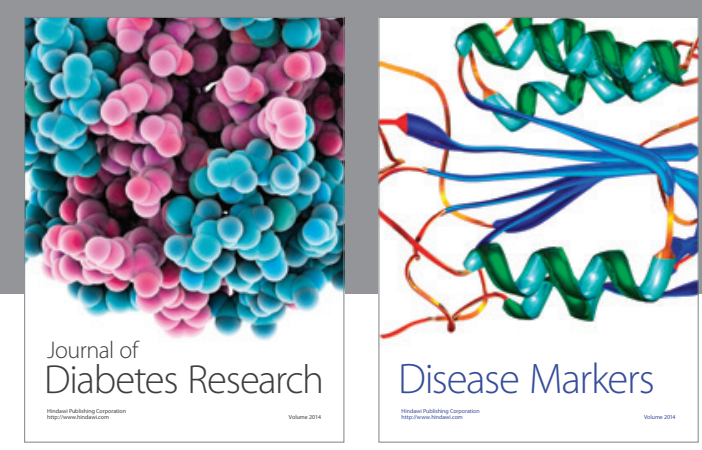

Disease Markers
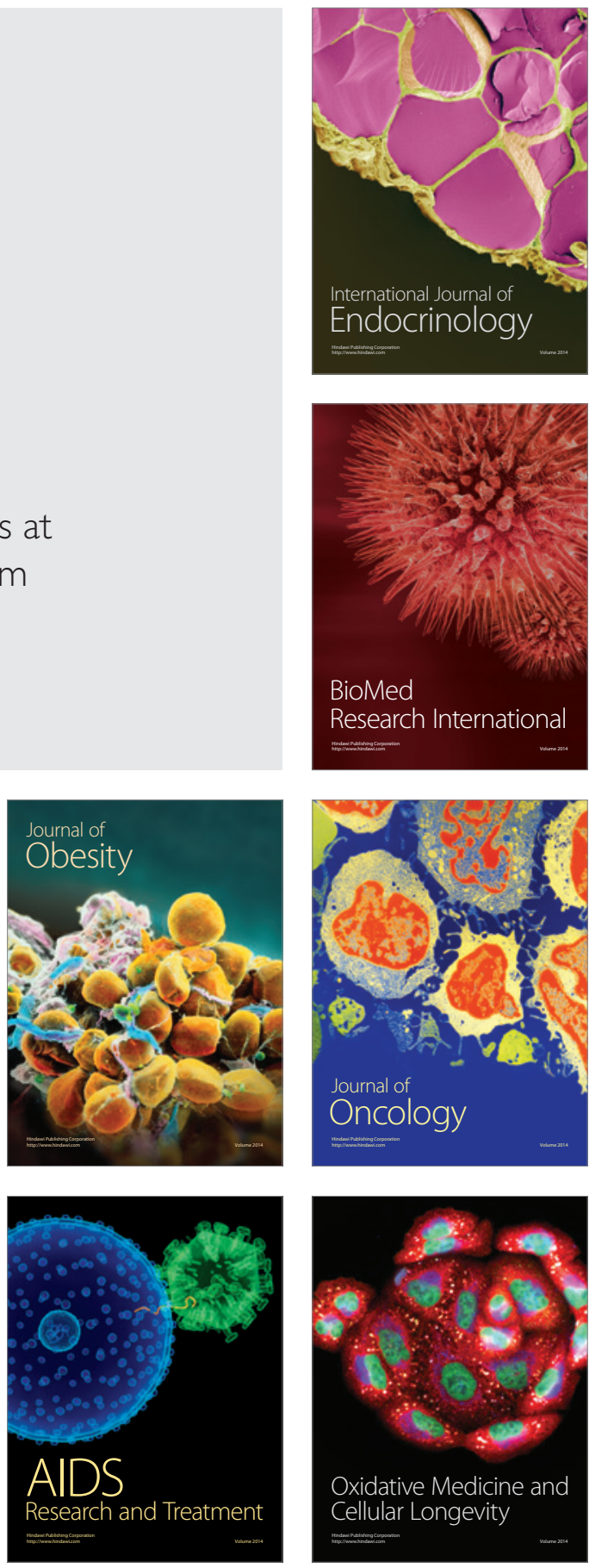